\title{
CONTROL OF VALVE CONVERTERS WITH NATURAL CURRENT CHARACTERISTICS
}

\author{
Sergazy Mendybayev, Yermek Sarsikeyeva, Dameli Kapanova \\ Kazakh Agro Technical University named after S. Seifullin, 010000, Astana, Kazakhstan
}

\begin{abstract}
The article considers the valve converter with a natural current characteristic, which is called the current of parametric, for which the primary is not the source of the electric force but a parametric source of current, to the output of which connected to the counter-parallel thyristor sets assembled on the bridge scheme. The control of thyristors in the current converter is carried out taking into account the characteristic features of the parametric source, to avoid overvoltage on its reactive elements, analyzed algorithmic and modulating functions of the system borne temporary diagrams.
\end{abstract}

\section{Introduction}

One of the most important tasks of modern production is energy saving, which is largely determined by the quality of electrical energy. The quality of electrical energy depends essentially on the type of harmonic composition of the current consumed. The harmonic composition of the network current is essentially dependent on the semiconductor transducers, which is a non-linear load for the supply network, which leads to significant distortion of the form of the network current and, as a consequence, deterioration of quality indicators electricity [1-4].

\section{Main part}

One of the ways to solve this problem is the application of the current parametric converters, which have a natural current characteristic. In the current of parametric, in contrast to traditional, primary is the source of voltage, and from the parametric source (PS), to which output the controlled valve sets (VS) are connected. The scheme of one of the variants of the bridge controlled CPC is shown in Figure 1.

\footnotetext{
${ }^{a}$ Corresponding author: sarsikeyev.ermek@yandex.ru
} 


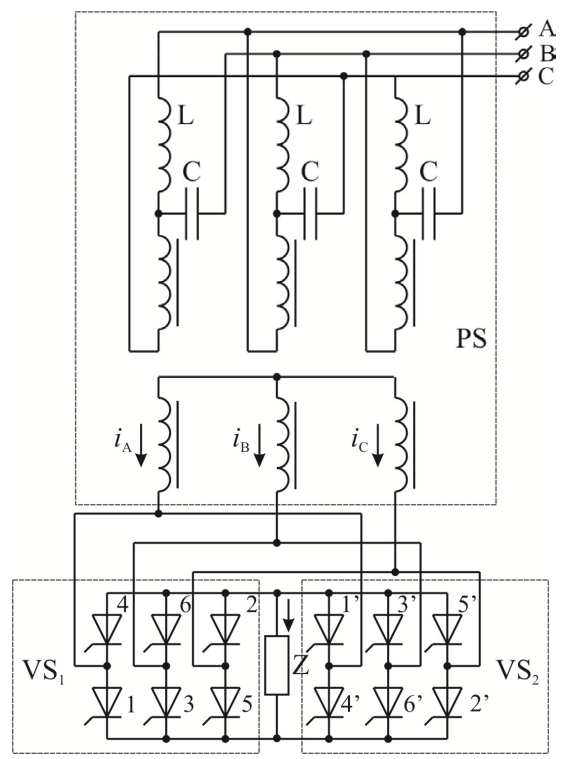

Fig. 1. Power scheme of CPC.

The control of thyristors in the CPC should take into account the following characteristics of the PSC $[5,6]$ :

-Necessity of presence of conducting valves at every moment in all phases of CPC, in order to avoid occurrence of surges on reactive elements of the current source;

-When unlocking the valves in a circuit with a voltage other than zero, there may be undesirable modes in which the thyristors are discharged capacitors, in circuits with almost zero resistance.

The main functions of the current converter control system Highlands The peculiarities of its operation. The control systems of the $\mathrm{CPC}$ should convert the set of input control and auxiliary signals into the control signals of thyristors.

The control signals can be digital and analogue. The control signals synchronized by the clock generator shall ensure the unlocking of the valves in the sequence necessary for the formation of the output current, the voltage of the required form-algorithmic function

The valve unlocking should occur at the moment of natural switching in order to avoid unfavorable transition processes. At the same time, the control signals should be such as to ensure the reliable inclusion of the valves in the case of breaking currents, having a minimum average control capacity $\left(R_{u}=R_{u \text { min }}\right)$.

The algorithmic function of the control system is implemented in a logical device, and it can be performed in the form of a machine or combinational logic schemes. The logical device, depending on input information coming to its input, sets the following modes of operation of the converter:

- Positive load current $\left(I_{\mathrm{H}}+\right)$;

- Negative current $\left(I_{\mathrm{H}^{-}}\right)$;

- Pause at Open load $\left(I_{\mathrm{H}}=0\right)$;

Modulating function of the control system of the CCI is realized in the device numberpulse control and performs modulation of temporal parameters of control effects:

-duration of pulses of control on thyristors of the current of parametric synchronized with the moment of natural unlocking of valves;

-output frequency converter;

-regulation of the output current by changing the whole number of cycles of pause. 
The generalized structure diagram of the device the number-pulse control is shown in Figure 2.

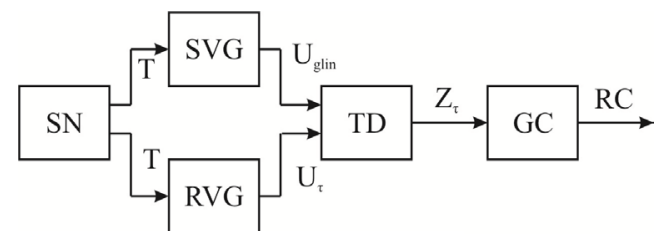

Fig. 2. Structural scheme of the device number-pulse control.

Sawtooth voltage is formed in the block of the sawtooth voltage generator (SVG). This synchronized voltage is compared with the support, coming from the output of the reference voltage generator (RVG), in the threshold device (TD) and at the moment of comparison at the output of the code generator (GC) the mode of operation of the converter changes.

In the threshold device, at the moment of comparison of these signals, the impulse which influencing on the code generator changes the mode of operation of the converter.

Analytical output signal device number-pulse regulation $\mathrm{Z}(\mathrm{t})$ is described:

$$
\begin{aligned}
& Z(t)=m, \text { at } t \leq t_{\text {response }} \\
& Z(t)=n, \text { at } t \geq t_{\text {response }}
\end{aligned}
$$

where $\mathrm{m}$ is the code $Z(t)$ before the threshold device is triggered; $n$ - code $Z(t)$ after the threshold device is triggered; $t_{\text {response }}$ - - response time of the threshold device.

The synchronization node is a sensor of moments of natural unlocking of valves. As such can be the current sensors included in the output phases of the parametric source, voltage sensors, controlling the moment of transition of the voltage "anode-cathode" valves through zero. Let us consider modulation of temporal parameters for nonreversible and reversible CPC.

1. Nonreversible current parametric converters. In Figure 3 there are temporary diagrams explaining the work of DNIC, where it is indicated: $\tau_{1}$-the duration of current flow in the load; $\tau_{2}$ - duration of a noncurrent pause; $U_{\tau 2}-$ the value of the reference voltage, which specifies the duration of the $\tau_{2} ; T_{2}$ - period of the output current period.

Up to the time of $t_{3}$, the load has a straightened current, corresponding to the combination of code words at the output of the code generator $-10\left(Z_{\tau l}\right)$.

At the moment of time $t_{3}$ voltage $U_{\text {glay }}=U_{\tau 2}$, as a result there is a response of TD and on output of the code generator there is a signal $Z_{\tau 2}$ corresponding to code 11 . This code determines the duration of the noncurrent pause. With change of $U_{\tau 2}$ changes $Z_{\tau 2}$, thus the average value of a load current is regulated.

The following variants of work are possible:

a) $\tau_{1}+\tau_{2}=T_{2}=$ const. With the change of the reference voltage $U_{\tau}$, simultaneously change the duration of signals $Z_{\tau 1}$ and $Z_{\tau 2}$

The period of the output current remains constant and is set by the period of quantization of the sawtooth voltage generator (Figure 3). The device number-pulse control has one channel containing a sawtooth generator and a reference voltage with one threshold device.

b) $\tau_{1}+\tau_{2}=T_{2}=v a r$. In this case, in turn, the following options are possible:

$\tau_{1}=$ const, $\tau_{2}=v a r$. The duration of the straightened current $\tau 1$ remains constant and is set by the reference voltage $U_{\tau l}=$ const. The duration of the noncurrent pause $\tau_{2} 2$ is a function of time and is determined by the reference voltage $U_{\tau 2}=v a r$, T.e. $\tau_{2}=f\left(U_{\tau 2}\right) . \tau_{1}=v a r$, $\tau_{2}=$ const. The duration of the straightened current $\tau_{1}(t)=f\left(U_{\tau l}\right), U_{\tau l}=$ var. The duration of the noncurrent pause remains constant and is set by the reference voltage $U_{\tau 2}=$ const. $\tau_{1}=$ var, $\tau_{2}=v a r$. The duration of the straightened current and the non-current pause are the 
functions of time and vary according to the change in the support voltage $U_{\tau 1}$ и $U_{\tau 2}=v a r$, $\tau_{1}(t)=f\left(U_{\tau l}\right), \tau_{2}=f\left(U \tau_{2}\right)$.

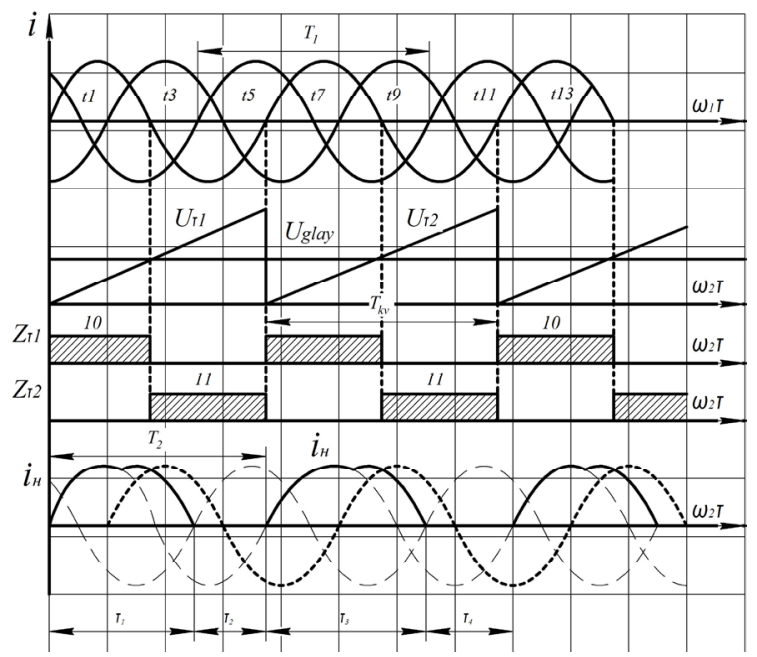

Fig. 3. Temporary diagrams of the device operation number-pulse regulation of the nonreversible CPC.

The device number - pulse control in the second variant has two channels, each of which contains a sawtooth voltage generator, a reference voltage generator and a threshold device. 2. Reversible currents parametric converters. In reversible current parametric converters are possible four time intervals on the period of the output current.

The device's temporary diagrams of the number-pulse control are shown in Figure 4, where indicated:

$\tau_{1}$ - the duration of the positive current $\left(I_{H}+\right)$;

$\tau_{3}$ - the duration of negative current $\left(I_{H}-\right)$;

$\tau_{2}$ - the duration of the noncurrent pause at transition from $I_{H}+$ to $I_{H^{-}}$;

$\tau_{4}$ - the duration of the noncurrent pause during the transition from $I_{H^{-}} \kappa I_{H^{+}}$.

$10-Z_{\tau l}$ signal code, specified by in + mode $I_{H}+$;

11 - signal code $Z_{\tau l a n d}$ и $Z_{\tau 2}$, that specify in $I_{H}=0$;

01 - signal code $Z_{\tau 3}$ setting mode $I_{H^{-}}$.

At the same time duration of intervals $\tau_{1} \div \tau_{4}$ two variants are possible:

a) dependent on each other;

b) independent of each other.

In the first variant the modulator has one generator of sawtooth voltage and two generators of reference voltages $\left(U_{\tau 1}, U_{\tau 2}\right)$, with corresponding threshold devices.

The duration of the signals $Z \tau_{1}$ are determined by the values of the reference voltage $U \tau_{1}$. Analytically this can be expressed as follows:

$$
\begin{aligned}
Z_{\tau 1} & =f\left(U_{\tau 1}\right), \\
Z_{\tau 2}=Z_{\tau 4} & =f\left(U_{\tau 3}-U_{\tau 4}\right), \\
Z_{\tau 3} & =f\left(U_{\tau 3}\right) .
\end{aligned}
$$

In the second case the modulator has four independent channels, each of which contains generators of sawtooth and reference voltages with a threshold device.

$$
Z_{\tau 1}=f\left(U_{\tau 1}\right), Z_{\tau 2}=f\left(U_{\tau 2}\right), Z_{\tau 3}=f\left(U_{\tau 3}\right), Z_{\tau 4}=f\left(U_{\tau 4}\right) .
$$




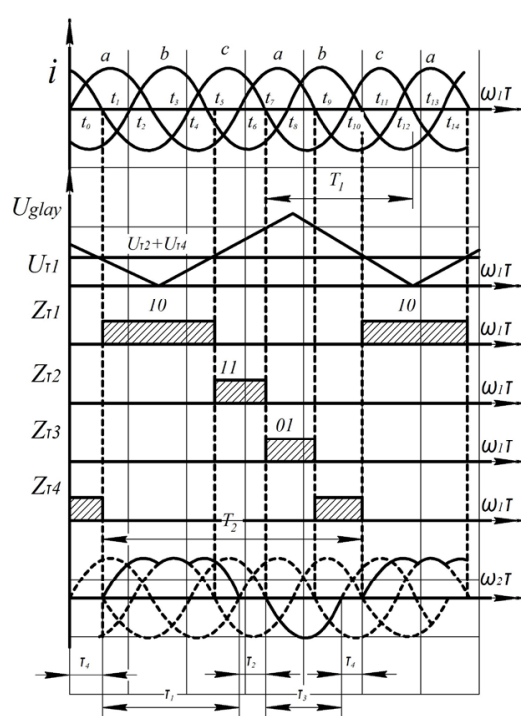

Fig. 4. Temporary diagrams of the device operation number-pulse regulation of reverse chamber with independent regulation.

\section{Conclusion}

Thus, the considered basic functions of the control system of CPC can be used at practical design of valve converters with a natural current characteristic for the most energyintensive consumers, which allows to improve the quality of electric power and reduce the negative impact of non-linear load on the electric network.

\section{References}

[1] B. Lukutin, Y. Sarsikeyev, M. Surkov, D. Lyapunov, Proceedings of 14th International Conference on Environment and Electrical Engineering, EEEIC, 6835913(2014)

[2] A. Petrusev, V. Rulevskiy, Ye. Sarsikeyev, D. Lyapunov, Proceedings of 2nd International Conference on Industrial Engineering, Applications and Manufacturing, ICIEAM, 7911461 (2016)

[3] N. V. Chicherina, V. Kazakov, O. Yu. Korneva, E. A. Titenko, The European Proceedings of Social \& Behavioural Sciences (EpSBS) 19, 349 (2017) doi:10.15405/epsbs.2017.01.47

[4] S.G. Obukhov, Ye.Zh. Sarsikeyev, I.I. Sholokhova, Proceedings of 2nd International Conference on Industrial Engineering, Applications and Manufacturing, ICIEAM, 7911454 (2016)

[5] K. Suker Power Electronics. Developer's Guide (Moscow, 2007).

[6] N. V. Chicherina, V. A. Tolkacheva, European Proceedings of Social \& Behavioural Sciences (EpSBS) 19, 683 (2017) doi:10.15405/epsbs.2017.01.90 